\title{
Nursing Management Plan for Children Suffering from Intestinal Parasites in Port Said Primary Care Centers
}

\author{
Hanaa Mohammed Ebrahim Nassar, Wafaa Elsayed Abd EL Gileel, \\ Amal Ahmed Khalil, Eman Moustafa EL-Hamshary \\ Department of , pediatric health nursing-Faculty of Nursing, Port Said University, \\ Department of, Pediatric health Nursing-Faculty of Nursing Ain Shams University, \\ Department of , pediatric health nursing-Faculty of Nursing, Port Said University, \\ Department of Parasitology- Faculty of Medicine, Suez Canal University
}

\begin{abstract}
Background: Intestinal parasitic infection in children is one of the most urgent problems during toddler and preschool stage of life. This period represents a challenge to the family and community and need greater attention to deal with this big problem. Nursing management plan include health education is important to improve the mother's knowledge and hygienic practices to prevent intestinal parasitic infection. .Aim: This study aimed at designing, implementing and evaluating the effect of a nursing management plan (NMP) for children who suffer from intestinal parasitic infection and their mothers at primary health care centers. Subject and Method: A sample of $\mathbf{2 3 0}$ children are from one to five years suffering from intestinal parasites infection and their mothers in study settings and 230 control. Conclusion: The study was conducted at seven primary health care centers in Port Said city. Data were collected usingchildren's medical record, pre designed questionnaire for mothers, an interviewing questionnaire for nurses and Likert types rating scale for children's attitude. Results: The results of the present study indicated that, E. histolytica and E. vermicularis were the most common intestinal parasitic infection in children in both study and control groups. According to the mother's knowledge, it was found that all mother's knowledge highly improved among study group from pre to post implementation of NMP. As regards reported mothers' practice, more than half of mother's total practice in study and control groups have inadequate practice related to pre-implementation of NMP. On the other hand, more than half of study and control groups have adequate practice in relation to post implementation of NMP. Recommendations: The research recommended that a well-planned prevention and control program, taking into account the personal and environmental risk factors at the household and/or community level is a necessary and important measure for solving the problem, especially in such low-resource areas.
\end{abstract}

Key Words: Nursing Management Plan, Toddler and Pre-school Children and Intestinal Parasites Infections 


\section{INTRODUCTION}

Intestinal parasitic infections (IPIs) are still a serious public health problem in the world. Parasitic diseases represent a major cause of morbidity and mortality in childhood in most parts of the world. Hygiene and play habits make children especially vulnerable to parasitic infections $\boldsymbol{E l - M a s r y ~ e t ~ a . l , ~ ( 2 0 0 7 ) . ~ I t ~ i s ~ e s t i m a t e d ~ t h a t ~}$ 3.5 billion people are affected, and 450 million are ill as a result of these infections, the majority being children. The high prevalence in children is attributed to many factors, particularly the social and economic situation of the individuals, which is the important cause of the prevalence of intestinal parasites, in addition to environmental condition Tappe et al., (2008).

In Egypt, the national data on prevalence of infection among children is lacking. Common IPIs present in Egypt are Enterobius (E.) vermiculars and Hymenolepis (H.) nana. E. vermiculars is the most prevalent parasite, it infects up to one third of children worldwide, as children frequently spread the infection, and E. vermiculars is most prevalent in day-care centers and schools. G. lamblia, another protozoan disease, is acquired through contaminated water or food El-Sahn et al. , (2000).

Parasitic infestations have great impact on life quality of people all over the world especially in developing countries the prevalence of parasitic infections in a particular region depends not only on bioenvironmental situation, but also on social, economic and cultural conditions.In developing countries that are mainly situated in tropical areas. lack of access to health services, malnutrition, and poor sanitation, increase vulnerability to infection because of physiological and immunological reasons children are more susceptible to parasitic infestation Hazratitappeh et al, (2010) \& Borg and Ryan, (2010).

Health problems outcomes of IPIs include malnutrition, growth stunting, intellectual retardation, and cognitive as well as educational deficiencies. IPIs are often physically and intellectually compromised by anemia, leading to attention deficits, learning disabilities, school absenteeism and higher dropout rates. Recurrent childhood infections can result in increased susceptibility to liver fibrosis, bladder cancer and kidney failure in adulthood Karan et a.l, (2012). Hence, parasitic diseases represent a major cause of morbidity and mortality in childhood in most parts of the world, as parasites are endemic in many parts of the world with no specific area is spared World Health Organization., (2007) \&Remme., (2007).

Control and prevention of parasites depends on an adequate knowledge of interactions among factors such as human behavior, the environment and the life cycles of parasites. The nurse's most important function is preventive education of children and their families regarding hygiene and health habits. Hence, nursing has a major role in the management and prevention of intestinal parasitic infections. Their close relationships with mothers of infected children provide a very good opportunity for health education regarding prevention of infection and re-infection Savage., (2002)\& Fitzgerald., (2015). Recently, in some countries, health promotion and awareness campaigns have used influential individuals to persuade others to adopt 
new practices, but this strategy has yet to be applied to prevention and control of intestinal parasitic diseases Espino et al., (2011).

\section{AIM OF STUDY:}

The present study aimed at designing, implementing and evaluating the effect of a nursing management plan for children suffering from intestinal parasites at primary care centers.

\section{SUBJECTS AND METHODS}

\section{Research Design}

This study followed a quasi-experimental research design with pre-post assessment in order to measure the effect of a nursing management plan of parasitic infection on children and their mothers.

\section{Setting:}

The study was carried out in seven Primary Health Care (PHC) centers. These are namely El-Salam and bank Al-Eskan, El-Kuwait, Arab, El-Manakh, Omar Ibn-AlKattab, Al-Kabouty, and Port-Fouad health care centers at Port Said city.

\section{Sample:}

The subject includes 460 children from both sex (male andfemale). Their age ranged from 1-5 years and they were accompanying by their mothers (study 230 and control 230 children). Sample size was estimated using the equation for the difference between two proportions Schlesselman, (1982). Calculation through Epi-Info statistical software package indicated an estimated sample size of 219 subjects per group. After adjustment for a drop-out rate of approximately $10 \%$, the sample size was increased to 230 children and their accompanying mothers per group. A multistage stratified random sampling technique was used to recruit the study sample of children and their mothers.

\section{Where:}

$$
\mathrm{N}=\frac{2 \mathrm{pq}\left(\mathrm{z}_{\alpha 2} \cdot \mathrm{z}_{\beta}\right)^{2}}{\left(\mathrm{p}_{1}-\mathrm{p}_{2}\right)^{2}}
$$

$\mathbf{n}=$ sample size per group.

$\mathbf{p}=(\mathrm{p} 1+\mathrm{p} 2) / 2$

$\mathbf{q}=1-\mathrm{p}$

$\mathbf{z \alpha 2}=1.96$

$\mathbf{z} \boldsymbol{\beta}=0.84$

\section{Tools of data collection:}

One tool was used to collect data from children and their companying mothers .

Tool (1): Pre-designed questionnaire: This questionnaire was designed by the researcher based on relevant literature review. It was in the form of close-ended questions, written in an Arabic language and included the followings parts:

Part I : This part was concerned with the socio-demographic variables of children (child's age, sex, birth order, nursery attendance) and their mothers (mothers' age, 
education, job status, family residence, income.....etc.)and home environment of the studied children (water supply, toilet and municipal sewage disposal .....etc)

Part II : This part included open and closed-end questions aimed at assessing the mothers' knowledge about intestinal parasitic infection ( nature, types, sources,. ....etc) and open and closed-ended questions with the aim of evaluating the mothers' reported practices related to parasitic infection, it covered the area of home sanitation and personal hygiene, food sanitation and general hygiene.

\section{Pilot Study:}

A pilot study was carried out including $10 \%$ of the expected sample size of the study subjects. The purpose of this pilot study was to test the clarity of the questions and statements, feasibility and consistency of the tools. Based on the findings of the pilot study, the necessary modifications were made for example, omit the question related to canal water because Post Said city have no canal, modify some words (rewording) to be suitable for illiterate mothers to understand. The final forms of the study tools were prepared and the time needed for completing the tests was determined to be about 20 to 30 minutes at health center. Pilot subjects were excluded from the main study sample.

\section{Data collection:}

Official approval letters were obtained from the Dean of Faculty of Nursing, Port Said University to directors of health care centers inPort Said City. These letters include a permission to carry out the study and explainthe purpose and nature of the study. Interviews were conducted individually with children and their accompanying mothers after getting their oral consent to participate and after explaining to them the purpose of the study. They were reassured about the confidentiality of the obtained information. A pilot study was conducted before starting data collection on (10) mothers who was excluded from the sample.

\section{Field Work:}

The study was conducted through the following four phases: assessment, planning, implementation and evaluation.

\section{Assessment}

The aim of this phase was to identify the baseline knowledge and reported practice of mothers at homes regarding prevention and control of the intestinal parasitic infestations

\section{Planning}

The planning phase aimed at identifying and spotting the nursing management plan needs as regards prevention and control of the intestinal parasitic diseases

\section{Implementation :}

The nursing management plan was designed based upon actual needs of children suffering from intestinal parasitic infection and was implemented in the form of sessions of different duration according to the content and response of children and their accompanying mothers. The nursing management plan divided into four sessions, they discussed different types of intestinal parasites infections (definition, causes, clinical picture, signs and symptoms, investigation, health teaching and 
specific protection according to level of prevention, treatment and complication, the data collected in the period from June 2009 till November 2010.

\section{Evaluation :}

Assessment of the degree of improvement in mothers' knowledge and reported practice towards intestinal parasitic infections as an impact of the exposure to the nursing management plan was done through a post-test six months after the intervention using the same (pre/test) data collection tools.

\section{Data Analysis:}

Scoring: A correct response was scored 1 and the in-correct scored zero for each item. For every one area of knowledge, the scores of the items were summed up and the total score divided by the number of the items, giving a mean score for the part. These scores were converted into a percent score. Knowledge was considered satisfactory if the percent score was $50 \%$ or more and unsatisfactory if less than 50\%.Mother reported practices composed three categories ( $0=$ sometimes; $1=$ usually; $2=$ always $)$. mother's practices were considered adequate if the percent score was $50 \%$ or more and inadequate if less than $50 \%$

Data entry and statistical analysis were done using SPSS 16.0 statistical software package. Qualitative categorical variables were compared using chi-square test. Differences between groups was detected using the student's T-test for quantitative data for the significance of differences between means of two groups and one way analysis of variance (ANOVA) was used for the significance of difference between means of more than two groups. Pearson correlation analysis was used for assessment of the inter-relationships among quantitative variables. Statistical significance was considered at $\mathrm{P}$-value $<0.05$.

\section{RESULTS:}

Table (1): shows the socio-demographic characteristics of children in the study and control groups. The age of children More than half in study and control groups were between $3-5$ years old $(56.5 \%, 51.7 \%$ respectively). As regarding sex, females were $53.9 \%$ and males were $53.9 \%$.

Regarding child birth order, $54.3 \%$ and $59.6 \%$ of study and control groups were the second and third birth order. About one third of children in study and control groups already entered the nursery school (36.5\% and $34.3 \%$ respectively) and about half of them were at kindergarten one $(54.8 \%$ and $48.1 \%$ respectively).

Table (2): shows the socio-demographic characteristics of mothers in the study and control groups. It was found that, $50.4 \%$ of the mothers in study group were in the age group of 20 to less than 30 years old and $45.7 \%$ of the mothers in control group were in the age group of 30 to less than 40 years old. More than half of the mothers in the study and control groups were having secondary school $(51.3 \%$ and $50.9 \%$ respectively). The table also shows that, more than $60 \%$ of the mothers were housewives in both study and control groups (67.8\% and $60.4 \%$ respectively). 
Table (3): shows that, sanitary home environment, more than two-thirds of the study and control groups had unsanitary home environment $(66.1 \%$ and $60.9 \%$ respectively). While more than one third of study and control groups had sanitary home environment (33.9\% and $39.1 \%$ respectively).

Table (4): shows that more than half of study and control groups $(59.6 \%$ and $52.2 \%$ respectively) had E. histolytica, followed by $40.9 \%$ in the study group and $43.9 \%$ in the control group had E. vermicular is before implementation of NMP. Regarding post implementation of NMP, $16.5 \%$ of study and $26.5 \%$ of control groups had a positive result of intestinal parasites. There are statistically significant differences between study and control groups in post implementation of NMP in relation to E. vermicular is and total positive result of intestinal parasites $(\mathrm{P}=0.03$ and 0.01 respectively).

Table (5): illustrates an improvement in mothers' knowledge regarding intestinal parasites post implementation of NMP compared with pre implementation results. There are statistically significant differences between study and control groups pre/post implementation of NMP.

Figure (1): illustrates the mother's total knowledge about intestinal parasites in the study and control groups pre-post implementation of NMP. As shown, about three-quarters $74.8 \%$ of mothers had unsatisfactory knowledge in the study and $74.8 \%$ of control groups pre implementation of NMP. Post implementation of NMP, the majority of mothers' knowledge in study group was improved (96.5\%), while the greater percentage of mother's knowledge in control group remained unsatisfactory (69.6\%). With statistical significant difference in relation to unsatisfactory total mother's knowledge post implementation of the NMP.

Table (6): displays that mother's practices in the study group pre implementation of NMP in home sanitation was $66.1 \%$, in personal hygiene $52.2 \%$, food sanitation $75.2 \%$ and general hygiene $49.6 \%$. This percentage increased to $70.9 \%, 53.5 \%, 77.4 \%$ and $57.8 \%$ respectively post implementation of NMP. Statistically significant differences were found between study and control groups pre implementation of NMP in relation to general hygienic practices $(\mathrm{P}=0.049)$.

Figure (2): illustrates that more than half of mothers had inadequate total reported practices in the study and control groups pre implementation $(54.3 \%$ and $53 \%$ respectively). Post implementation of NMP mothers had adequate practices regarding intestinal parasites in study and control groups (62.2\% and 53\% respectively). There is a statistically significant differences between study and control groups post implementation of NMP in relation to adequate total reported practices $(\mathrm{P}=0.048)$.

Table (7): displays that about two third of mothers $(65.7 \%)$ who had satisfactory knowledge their children hadn't intestinal parasites infection. On the other hand, less than half of mothers $(47.3 \%)$ who had adequate reported practices, their children hadn't intestinal parasites. Mothers who had unsatisfactory knowledge and inadequate 
reported practices, their children had intestinal parasites infection $(69.6 \%$ and $57.6 \%$ respectively). There is a statistically significant relation between total mothers' knowledge and diagnosis of intestinal parasites $(\mathrm{P}=<0.001)$.

Table (8): represents the correlation between the mothers' total knowledge and their total reported practices pre-post the implementation of NMP. The table demonstrates a statistically significant positive correlation between mothers' total knowledge and their total reported practices pre implementation of NMP in study and control groups ( $\mathrm{r}=168$ and 161 respectively). Also there is a positive correlation between mothers' total knowledge and their total reported practices in study group post implementation of NMP $(r=428)$.

Table (1): Socio-demographic characteristics of children

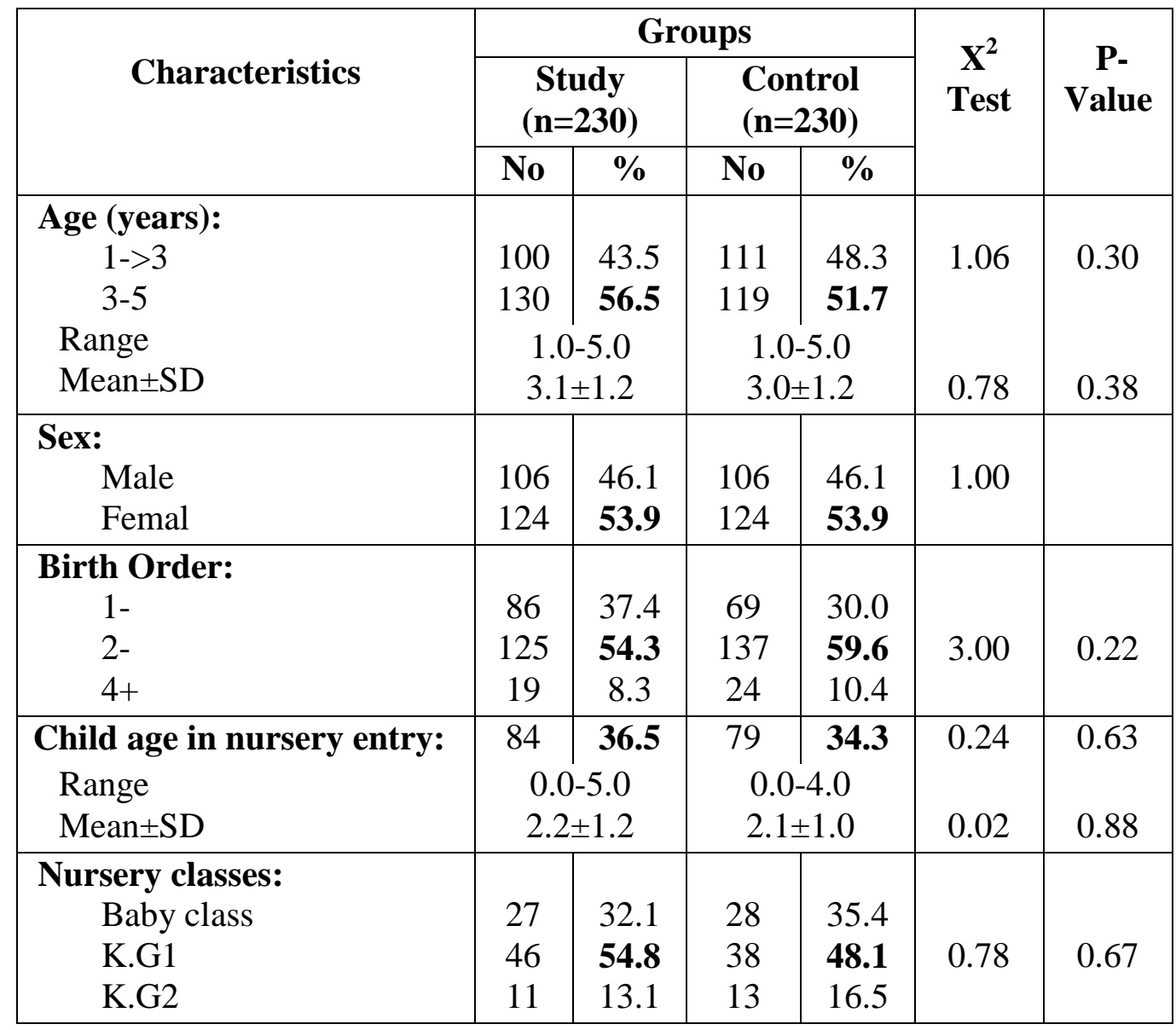

(*) Statistically significant at $p<0.05$ 
Table (2): Socio-demographic characteristics of mothers

\begin{tabular}{|c|c|c|c|c|c|c|}
\hline \multirow{3}{*}{$\begin{array}{l}\text { Socio-demographic } \\
\text { characteristics }\end{array}$} & \multicolumn{4}{|c|}{ Groups } & \multirow{3}{*}{$\begin{array}{c}\mathrm{X}^{2} \\
\text { Test }\end{array}$} & \multirow{3}{*}{ P-Value } \\
\hline & \multicolumn{2}{|c|}{$\begin{array}{c}\text { Study } \\
(\mathrm{n}=230)\end{array}$} & \multicolumn{2}{|c|}{$\begin{array}{l}\text { Control } \\
(\mathrm{n}=230)\end{array}$} & & \\
\hline & No & $\%$ & No & $\%$ & & \\
\hline $\begin{array}{l}\text { Mothers' age in years: } \\
20-\end{array}$ & 116 & 50.4 & 95 & 41.3 & & \\
\hline $30-$ & 99 & 43.0 & 105 & 45.7 & 7.27 & $0.03^{*}$ \\
\hline $40+$ & 15 & 6.5 & 30 & 13.0 & & \\
\hline Education: & & & & & & \\
\hline Illiterate & 19 & 8.3 & 20 & 8.7 & & \\
\hline Read/write & 13 & 5.7 & 12 & 5.2 & & \\
\hline Primary & 12 & 5.2 & 8 & 3.5 & 3.16 & 0.68 \\
\hline Preparatory & 18 & 7.8 & 12 & 5.2 & & \\
\hline Secondary & 118 & 51.3 & 117 & 50.9 & & \\
\hline University & 50 & 21.7 & 61 & 26.5 & & \\
\hline Job Status: & & & & & 73 & 0 \\
\hline $\begin{array}{l}\text { Housewife } \\
\text { Working }\end{array}$ & $\begin{array}{c}156 \\
74\end{array}$ & $\begin{array}{l}67.8 \\
32.2\end{array}$ & $\begin{array}{c}139 \\
91\end{array}$ & $\begin{array}{l}\mathbf{6 0 . 4} \\
39.6\end{array}$ & 3 & 0.10 \\
\hline
\end{tabular}

(*) Statistically significant at $p<0.05$

Table (3): Home characteristics as reported by mothers

\begin{tabular}{|c|c|c|c|c|c|c|}
\hline \multirow{3}{*}{ Home facilities } & \multicolumn{4}{|c|}{ Groups } & \multirow{3}{*}{$\begin{array}{c}\mathbf{X}^{2} \\
\text { Test }\end{array}$} & \multirow{3}{*}{$\begin{array}{c}\text { P- } \\
\text { Value }\end{array}$} \\
\hline & \multicolumn{2}{|c|}{$\begin{array}{c}\text { Study } \\
(n=230)\end{array}$} & \multicolumn{2}{|c|}{$\begin{array}{l}\text { Control } \\
(n=230)\end{array}$} & & \\
\hline & No & $\%$ & No & $\%$ & & \\
\hline Each room has a window & 212 & 92.2 & 221 & 96.1 & 3.19 & 0.07 \\
\hline Separate kitchen & 219 & 95.2 & 220 & 95.7 & 0.05 & 0.82 \\
\hline Gas stove & 226 & 98.3 & 227 & 98.7 & Fisher & 1.00 \\
\hline Fridge & 225 & 97.8 & 224 & 97.4 & 0.09 & 0.76 \\
\hline Electricity & 229 & 99.6 & 230 & 100 & Fisher & 1.00 \\
\hline Water supply & 225 & 97.8 & 224 & 97.4 & 0.09 & 0.76 \\
\hline Water closet & 229 & 99.6 & 227 & 98.7 & Fisher & 0.62 \\
\hline House is sunny & 198 & 86.1 & 190 & 82.6 & 1.05 & 0.30 \\
\hline Municipal sewage disposal & 213 & 92.6 & 218 & 94.8 & 0.92 & 0.34 \\
\hline No pets at home & 188 & 81.7 & 192 & 83.5 & 0.24 & 0.62 \\
\hline Separate place for pets (if present) & 32 & 76.2 & 24 & 63.2 & 1.61 & 0.20 \\
\hline \multicolumn{7}{|l|}{ Total Home Environment } \\
\hline Sanitary & 78 & 33.9 & 90 & 39.1 & & \\
\hline Unsanitary & 152 & 66.1 & 140 & 60.9 & 1.35 & 0.25 \\
\hline
\end{tabular}

(*) Statistically significant at $p<0.05$ 
Table (4): Distribution of the studied sample according to their diagnosis pre-post Implementation of NMP.

\begin{tabular}{|c|c|c|c|c|c|c|c|c|c|c|}
\hline \multirow[t]{3}{*}{ Diagnosis } & \multicolumn{4}{|c|}{ Pre-Implementation } & \multicolumn{4}{|c|}{$\begin{array}{c}\text { Post- } \\
\text { Implementation }\end{array}$} & \multirow{3}{*}{$\begin{array}{c}\mathbf{X}^{2} \text { Test } \\
(p- \\
\text { value }) \\
\text { Pre }\end{array}$} & \multirow{3}{*}{$\begin{array}{c}\mathbf{X}^{2} \text { test } \\
\text { (P- } \\
\text { Value) } \\
\text { Post }\end{array}$} \\
\hline & \multicolumn{2}{|c|}{$\begin{array}{c}\text { Study } \\
(n=230)\end{array}$} & \multicolumn{2}{|c|}{$\begin{array}{l}\text { Control } \\
(n=230)\end{array}$} & \multicolumn{2}{|c|}{$\begin{array}{c}\text { Study } \\
(n=230)\end{array}$} & \multicolumn{2}{|c|}{$\begin{array}{l}\text { Control } \\
(n=230)\end{array}$} & & \\
\hline & No & $\%$ & No & $\%$ & No & $\%$ & No & $\%$ & & \\
\hline $\begin{array}{l}\text { A. } \\
\text { lumbricoides }\end{array}$ & 20 & 8.7 & 18 & 7.8 & 1 & 0.4 & 2 & 0.9 & $\begin{array}{l}0.11 \\
0.73\end{array}$ & $\begin{array}{c}\text { Fisher } \\
1.00\end{array}$ \\
\hline Ancylostoma & 3 & 1.3 & 7 & 3.0 & 0 & 0.0 & 1 & 0.4 & $\begin{array}{c}\text { Fisher } \\
0.18\end{array}$ & $\begin{array}{c}\text { Fisher } \\
1.00\end{array}$ \\
\hline E. histolytica & 137 & $\begin{array}{c}59 . \\
6\end{array}$ & 120 & $\begin{array}{c}52 . \\
2\end{array}$ & 27 & $\begin{array}{c}11 . \\
7\end{array}$ & 36 & $\begin{array}{c}15 . \\
7\end{array}$ & $\begin{array}{l}2.55 \\
0.11\end{array}$ & $\begin{array}{l}1.49 \\
0.22\end{array}$ \\
\hline $\begin{array}{l}\text { E. } \\
\text { vermicularis }\end{array}$ & 94 & $\begin{array}{c}40 . \\
9\end{array}$ & 101 & $\begin{array}{c}43 . \\
9\end{array}$ & 12 & 5.2 & 25 & $\begin{array}{c}10 . \\
9\end{array}$ & $\begin{array}{l}0.44 \\
0.51\end{array}$ & $\begin{array}{c}4.97 \\
0.03 *\end{array}$ \\
\hline G. lamblia & 3 & 1.3 & 5 & 2.2 & 0 & 0.0 & 0 & 0.0 & $\begin{array}{c}\text { Fisher } \\
0.45\end{array}$ & $\begin{array}{l}0.00 \\
1.00\end{array}$ \\
\hline H. nana & 3 & 1.3 & 1 & 0.4 & 0 & 0.0 & 0 & 0.0 & $\begin{array}{c}\text { Fisher } \\
1.00\end{array}$ & $\begin{array}{l}0.00 \\
1.00\end{array}$ \\
\hline S. mansoni & 2 & 0.9 & 4 & 1.7 & 0 & 0.0 & 0 & 0.0 & $\begin{array}{c}\text { Fisher } \\
0.69\end{array}$ & $\begin{array}{l}0.00 \\
1.00\end{array}$ \\
\hline Total positive & 230 & $\begin{array}{c}100 \\
.0\end{array}$ & 230 & $\begin{array}{c}100 \\
.0\end{array}$ & 38 & $\begin{array}{c}16 . \\
5\end{array}$ & 61 & $\begin{array}{c}26 . \\
5\end{array}$ & $\begin{array}{c}\text { Fisher } \\
0.25\end{array}$ & $\begin{array}{c}6.81 \\
0.01 *\end{array}$ \\
\hline
\end{tabular}

(*) statistically significant at $p<0.05$ 
Table (5): Mother's knowledge about intestinal parasites pre-post implementation of NMP.

\begin{tabular}{|c|c|c|c|c|c|c|c|c|c|c|}
\hline \multirow[t]{3}{*}{$\begin{array}{c}\text { Mother's } \\
\text { Knowledge }\end{array}$} & \multicolumn{4}{|c|}{ Pre-Implementation } & \multicolumn{4}{|c|}{$\begin{array}{c}\text { Post- } \\
\text { Implementation }\end{array}$} & \multirow{3}{*}{$\begin{array}{c}\mathrm{X}^{2} \text { Test } \\
(\mathrm{p}- \\
\text { value }) \\
\text { Pre }\end{array}$} & \multirow{3}{*}{$\begin{array}{c}\mathrm{X}^{2} \text { Test } \\
(\mathbf{P}- \\
\text { Value }) \\
\text { Post }\end{array}$} \\
\hline & \multicolumn{2}{|c|}{$\begin{array}{c}\text { Study } \\
(\mathbf{n}=230)\end{array}$} & \multicolumn{2}{|c|}{$\begin{array}{l}\text { Control } \\
(\mathbf{n}=230)\end{array}$} & \multicolumn{2}{|c|}{$\begin{array}{c}\text { Study } \\
(n=230)\end{array}$} & \multicolumn{2}{|c|}{$\begin{array}{l}\text { Control } \\
(\mathbf{n}=230)\end{array}$} & & \\
\hline & No & $\%$ & No & $\%$ & No & $\%$ & No & $\%$ & & \\
\hline Nature & 150 & $\begin{array}{c}65 . \\
2\end{array}$ & 129 & $\begin{array}{c}65 . \\
1\end{array}$ & 226 & $\begin{array}{c}98 . \\
3\end{array}$ & 152 & $\begin{array}{c}66 . \\
1\end{array}$ & $\begin{array}{c}4.02 \\
\left(0.045^{*}\right)\end{array}$ & $\begin{array}{c}81.27 \\
(<0.001 \\
*)\end{array}$ \\
\hline Types & 31 & $\begin{array}{c}13 . \\
5\end{array}$ & 40 & $\begin{array}{c}17 . \\
4\end{array}$ & 216 & $\begin{array}{c}93 . \\
9\end{array}$ & 47 & $\begin{array}{c}20 . \\
4\end{array}$ & $\begin{array}{c}1.35 \\
(0.05)\end{array}$ & $\begin{array}{c}253.58 \\
(<0.001 \\
*)\end{array}$ \\
\hline Sources & 49 & $\begin{array}{c}21 . \\
3\end{array}$ & 53 & $\begin{array}{c}23 . \\
0\end{array}$ & 208 & $\begin{array}{c}90 . \\
4\end{array}$ & 64 & $\begin{array}{c}27 . \\
8\end{array}$ & $\begin{array}{c}0.20 \\
(0.65)\end{array}$ & $\begin{array}{c}186.53 \\
(<0.001 \\
*)\end{array}$ \\
\hline Manifestations & 63 & $\begin{array}{c}27 . \\
4\end{array}$ & 74 & $\begin{array}{c}32 . \\
2\end{array}$ & 218 & $\begin{array}{c}94 . \\
8\end{array}$ & 90 & $\begin{array}{c}39 . \\
1\end{array}$ & $\begin{array}{c}1.26 \\
(0.26)\end{array}$ & $\begin{array}{c}160.98 \\
(<0.001 \\
*)\end{array}$ \\
\hline Diagnosis & 38 & $\begin{array}{c}16 . \\
5\end{array}$ & 52 & $\begin{array}{c}22 . \\
6\end{array}$ & 202 & $\begin{array}{c}87 . \\
8\end{array}$ & 60 & $\begin{array}{c}26 . \\
1\end{array}$ & $\begin{array}{c}2.71 \\
(0.10)\end{array}$ & $\begin{array}{c}178.80 \\
(<0.001 \\
*)\end{array}$ \\
\hline Prevention & 134 & $\begin{array}{c}58 . \\
3\end{array}$ & 147 & $\begin{array}{c}63 . \\
9\end{array}$ & 220 & $\begin{array}{c}95 . \\
7\end{array}$ & 154 & $\begin{array}{c}67 . \\
0\end{array}$ & $\begin{array}{c}1.55 \\
(0.21)\end{array}$ & $\begin{array}{c}62.30 \\
(<0.001 \\
*)\end{array}$ \\
\hline Role of health center & 110 & $\begin{array}{c}47 . \\
8\end{array}$ & 114 & $\begin{array}{c}49 . \\
6\end{array}$ & 216 & $\begin{array}{c}93 . \\
9\end{array}$ & 127 & $\begin{array}{c}55 . \\
2\end{array}$ & $\begin{array}{c}0.14 \\
(0.71)\end{array}$ & $\begin{array}{c}90.79 \\
(<0.001 \\
*)\end{array}$ \\
\hline Complication & 79 & $\begin{array}{c}34 . \\
3\end{array}$ & 90 & $\begin{array}{c}39 . \\
1\end{array}$ & 218 & $\begin{array}{c}94 . \\
8\end{array}$ & 92 & $\begin{array}{c}40 . \\
0\end{array}$ & $\begin{array}{l}1.13 \\
(0.29)\end{array}$ & $\begin{array}{c}157.05 \\
(<0.001 \\
*)\end{array}$ \\
\hline
\end{tabular}

(*) Statistically significant at $p<0.05$ 

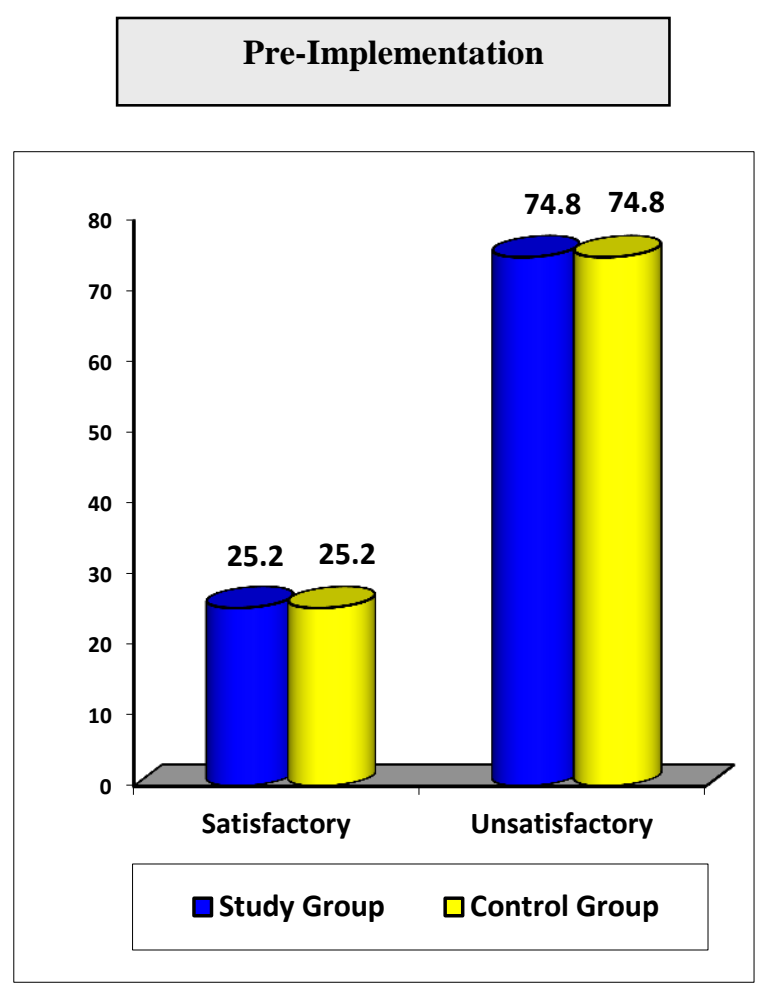

\section{Post-Implementation}

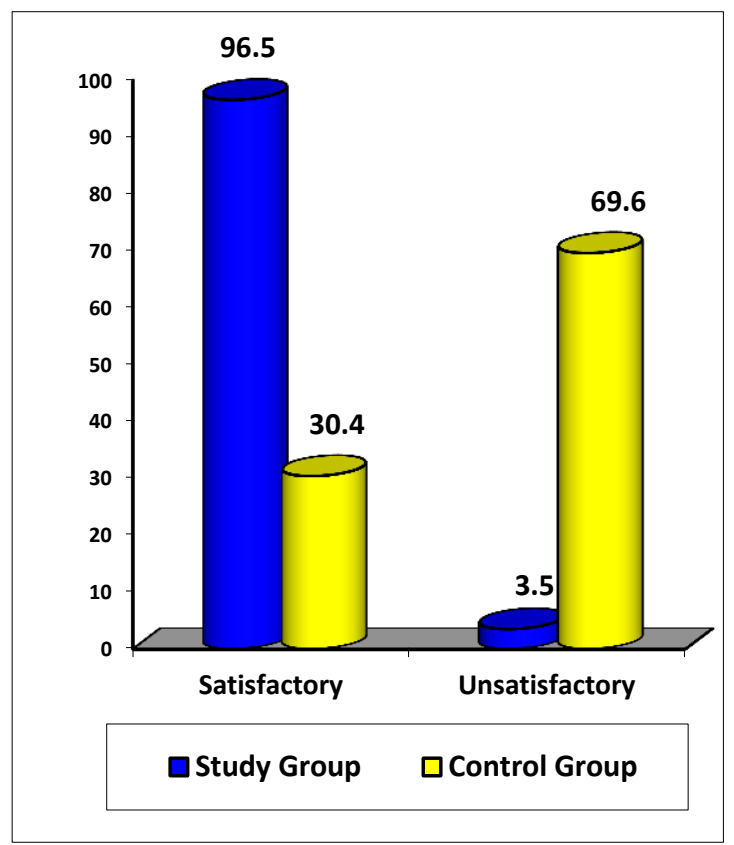

Figure (1): Total mother's knowledge about intestinal parasites pre-post implementation of NMP.

Table (6): Mothers' reported practices pre-post implementation of NMP.

\begin{tabular}{|c|c|c|c|c|c|c|c|c|c|c|}
\hline \multirow[t]{3}{*}{ Total practices } & \multicolumn{4}{|c|}{ Pre- Implementation } & \multicolumn{4}{|c|}{$\begin{array}{c}\text { Post- } \\
\text { Implementation }\end{array}$} & \multirow{3}{*}{$\begin{array}{c}\mathrm{X}^{2} \text { Test } \\
\text { (P- } \\
\text { Value) } \\
\text { Pre }\end{array}$} & \multirow{3}{*}{$\begin{array}{c}X^{2} \text { Test } \\
\text { (P- } \\
\text { Value) } \\
\text { Post }\end{array}$} \\
\hline & \multicolumn{2}{|c|}{$\begin{array}{c}\text { Study } \\
(n=230)\end{array}$} & \multicolumn{2}{|c|}{$\begin{array}{l}\text { Control } \\
(n=230)\end{array}$} & \multicolumn{2}{|c|}{$\begin{array}{c}\text { Study } \\
(n=230)\end{array}$} & \multicolumn{2}{|c|}{$\begin{array}{l}\text { Control } \\
(n=230)\end{array}$} & & \\
\hline & No & $\%$ & No & $\%$ & No & $\%$ & No & $\%$ & & \\
\hline Home sanitation & 152 & 66.1 & 151 & 65.7 & 163 & 70.9 & 156 & 67.8 & $\begin{array}{l}0.01 \\
0.92\end{array}$ & $\begin{array}{l}0.50 \\
0.48\end{array}$ \\
\hline Personal hygiene & 120 & 52.2 & 130 & 56.5 & 123 & 53.5 & 126 & 54.8 & $\begin{array}{l}0.88 \\
0.35\end{array}$ & $\begin{array}{l}0.08 \\
0.78\end{array}$ \\
\hline Food sanitation & 173 & 75.2 & 175 & 76.1 & 178 & 77.4 & 177 & 77.0 & $\begin{array}{l}0.05 \\
0.83\end{array}$ & $\begin{array}{l}0.01 \\
0.91\end{array}$ \\
\hline General hygiene & 114 & 49.6 & 93 & 40.4 & 133 & 57.8 & 119 & 51.7 & $\begin{array}{c}3.87 \\
0.049 *\end{array}$ & $\begin{array}{l}1.72 \\
0.19\end{array}$ \\
\hline
\end{tabular}

(*) Statistically significant at $p<0.05$ 

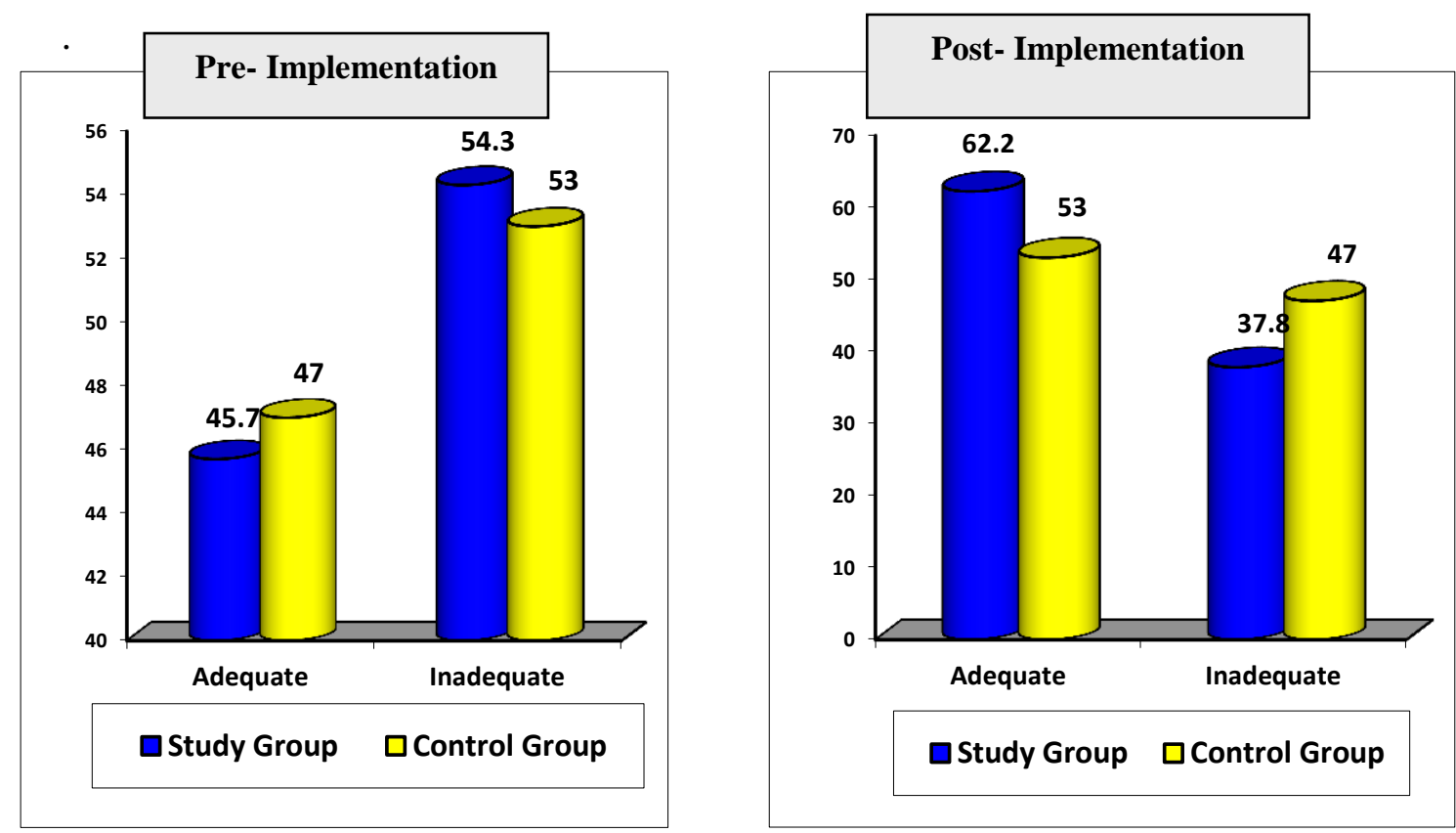

Figure (2): Total mothers' reported practices pre- post implementation of NMP.

Table (7): Relation between the mother's total knowledge and their total reported practices and lab finding as indictor of NMP success.

\begin{tabular}{|c|c|c|c|c|c|c|}
\hline \multirow{3}{*}{$\begin{array}{l}\text { Total knowledge and } \\
\text { reported Practices }\end{array}$} & \multicolumn{4}{|c|}{$\begin{array}{c}\text { lab result for Intestinal } \\
\text { Parasites }\end{array}$} & \multirow{3}{*}{$X^{2}$ Test } & \multirow{3}{*}{ P-Value } \\
\hline & \multicolumn{2}{|c|}{ Negative } & \multicolumn{2}{|c|}{ Positive } & & \\
\hline & No & $\%$ & No & $\%$ & & \\
\hline $\begin{array}{l}\text { Total Knowledge: } \\
\text { Satisfactory } \\
\text { Unsatisfactory }\end{array}$ & $\begin{array}{l}239 \\
125\end{array}$ & $\begin{array}{l}\mathbf{6 5 . 7} \\
34.3\end{array}$ & $\begin{array}{l}169 \\
387\end{array}$ & $\begin{array}{l}30.4 \\
\mathbf{6 9 . 6}\end{array}$ & 110.84 & $<0.001 *$ \\
\hline $\begin{array}{l}\text { Total reported Practice: } \\
\text { Adequate } \\
\text { Inadequate }\end{array}$ & $\begin{array}{l}172 \\
192\end{array}$ & $\begin{array}{l}\mathbf{4 7 . 3} \\
52.7\end{array}$ & $\begin{array}{l}236 \\
320\end{array}$ & $\begin{array}{l}42.4 \\
\mathbf{5 7 . 6}\end{array}$ & 2.06 & 0.15 \\
\hline
\end{tabular}

(*) statistically significant at $p<0.05$

Table (8): Correlation between mother's total knowledge and their total reported practices pre-post implementation of NMP.

\begin{tabular}{|l|c|c|c|c||c|}
\hline \multirow{3}{*}{$\begin{array}{c}\text { Total reported } \\
\text { Practice }\end{array}$} & \multicolumn{3}{|c|}{ Pearson correlation(knowledge score) } & \multirow{2}{*}{ Overall } \\
\cline { 2 - 5 } & \multicolumn{2}{|c|}{$\begin{array}{c}\text { Pre-Implementation - } \\
\text { Implementation }\end{array}$} & \multicolumn{2}{|c|}{$121^{* *}$} \\
\cline { 2 - 5 } & Study & Control & Study & Control & \\
\hline Practice & $168^{*}$ & $161^{*}$ & $428^{* *}$ & 060 & 100 \\
\hline
\end{tabular}




\section{DISCUSSION:}

The epidemiology of the intestinal infestations is a major health problem and estimated to affect 3.5 billion people, most of whom are children residing in developing countries $\boldsymbol{W H O ( 2 0 0 0 )}$. Nurses participate in intestinal parasitic prevention at several levels. Nurses have developed model prevention programs aimed at high risk children. They teach how to deal effectively with children Gross \& Grady, (2002). Nurses are in a prime position to identify children at risk by virtue of their work in primary care and health clinics, through these and future efforts, nurses are meaningful participants in intestinal parasitic prevention Cooper et al., (2000).

The present study showed that, more than two thirds of the study sample had unsanitary home environment. This may be due to the greater link between environmental condition and prevalence of intestinal parasites infection. The study finding is inan agreement with Sevig \& $\underline{\text { Kolac }}$ (2000)who concluded that, poor environmental conditions and poor personal hygiene influenced the acquisition of intestinal parasites.Celiksoz., et al (2005)added that, poor environmental conditions, low personal hygiene, lack of public water supplies and waste removal services accompanied with low socioeconomic condition increased the prevalence of intestinal parasites in children.

Most common intestinal parasite infections in the study sample were E. histolytica and E. Vermicularis. This could be due to poor personal hygiene, bad dietary habits and poor environment. After Applying nursing management plan and taking medication, significant improvement occurred in the study group. Also there was an improvement in the control group that could be due to compliance in taking medication but the improvement was statistically less than the study group. This result is supported byElMasry H.etal., (2007) whoreported that, E. vermicularis was the most common intestinal parasite seen in the primary care setting.Helminths were unusually high, E. histolytica, E. coli and E. nana occurred in 97\%, 98\% and 93\% of specimens respectively.

According to a study by Mwambete \& Kalison (2006) the most prevalent protozoan was E. histolytica detected in children. Also the study of Ayalew et al., (2011) clarified that, the rate of pathogenic protozoa was G. lamblia (10.9\%) followed by E. histolytica $(4.1 \%)$, the prevalence rate of nonpathogenic protozoan, E. coli was $(11.3 \%)$ in the children's stools and H. nana was $(3.0 \%)$ of the intestinal helminthes.

The lowest percentage of parasites in this study was Schistosomasis. This may be due to that Port Said city does not havesmall canals. In the same line Taylor M. (2008) found that, water contact activities represented a significant risk, children playing or swimming in canals had higher rate of infection and morbidity by Schistosomiasis. Also HallA. etal, (2008)reported that the most prevalent parasites were A. 
lumbricoides (35\%), G. lamblia (14\%) and Intestinal worms of which the nematodes and the hookworms are the most common parasites among children.

Globally in the findings of the present study the pre implementation of NMP, knowledge of mothers regarding parasites was unsatisfactory in both groups study and control. While the study group had greater improvement in knowledge post implementation of NMP. This may be due to the children's mothers not exposed to any

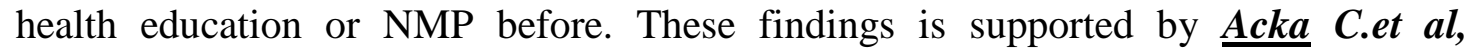
(2010)who reported thatbefore the implication for Integrated Control, knowledge of children's mothers regarding parasites was lacked. However, knowledge about intestinal parasite and related unhealthy habits significantly improved after the integrated control.

Regarding the mother's reported practicespre- post implementation of NMPas shown in (table, 6), more than half of mothers in study and control groups had inadequate reported practices pre implementation of NMP, while the mothers practices slightly improved post implementation of NMP. This improvement included home sanitation, personal hygiene, food sanitation and general hygiene. This may be due to the idea that these practicesare considered as normal habits and behaviors that need more modifications to improve it. From this perspective, $\underline{K a n o a ~} \boldsymbol{B}$. and $\underline{\text { Al-Hindi } \boldsymbol{A} \text {. }}$ (2009)stated that, health education programme was applied for 6 months only and this period was insufficient and the impact of health education will come on the long run (1 year to 5 year) .

Regarding total mothers' reported practices pre- post implementation of NMP,the findings of the present study (figure, 2) showed that, improvement was occurred in mother's total reported practices within study group post implementation of NMP due to acquiring new knowledge that help them in practices new health habits and behavior to deal with IPIs. This finding is supported by Vivas., et al (2010)who reported that, knowledge and attitude of mothers were moderate and the practice of mothers about intestinal parasitic infections of their children was fairly good. It is suggested that by education and attitude of mothers, it is possible to prevent intestinal parasitic infection. Azian., et al (2007) stated that, the high prevalence of intestinal parasites was attributed to poor environmental management, poor personal hygiene and lack of health education. The impact of regular health education in improving attitude and practices in the control of intestinal parasites was examined in four rural areas of Bangladesh. This result showed highly significant improvements in mother's practice regards intestinal parasitic infections.

The results in the present study revealed that, there was a statistically significant positive correlation between mother's knowledge and lab finding as indictor of NMP success of children's intestinal parasitic infections (table, 7). This result may be due to when mother's awareness increase about intestinal parasites infections such as mode of transmissions and methods of prevention that encourage mother try to prevent this infection away from their children so the incidence of IPIs was decreased. In the same 
line $\mathbf{T u} X \boldsymbol{X}$., et al (2003) revealed that, after the implementation of the intervention in the past decade, the total parasites prevalence declined significantly.

The present study showed a statistically significant positive correlation between mother's knowledge and practice pre and post implementation of NMP regarding study and control groups (table, 8). This finding points to the successful effect of the NMP about intestinal parasitic infection for children and their mothers.Inthe same line Curtale., et al (1998) cleared that, a good level of knowledge was observed for ways of preventing infection with mother practice. Vivas A.et al (2010)added that, proper knowledge of hygiene was associated with personal hygienic practice. According to the result finding of Amein., et al (2014) reported that, a highly statistically significant difference between the caregivers' and their knowledge and practice about intestinal parasites infection, also, the study found that significant improvement of knowledge, practices of caregivers regarding intestinal parasitic infestations.

\section{CONCOLUSION:}

Based on the findings of the present study it can be concluded that; Total mothers' knowledge was unsatisfactory in study and control group's pre implementation of NMP and become more satisfactory in the study group post implementation of NMP. More than half of mothers' total reported practice in study and control groups exhibited inadequate reported practice pre implementation of NMP. On the other hand, most of mothers in study group got adequate practice in post implementation of NMP.

\section{RECOMMENDATIONS:}

Based on the results of the present study, the researcher came up with the following recommendations: Increase public awareness about knowledge of intestinal parasitic infestation and its prevention by community leaders, mass media, and others especially at rural area. Improving personal and environmental hygienic measures and regular screening and treatment for parasitic infestation. More studies on big number of students in rural and urban areas at Egypt. Apply programmers to children from one year to five years should consider adding periodic anthelmintic treatments in health care centers. Periodic health education for children and their mothers to improve their knowledge and practice regarding intestinal parasites.

\section{REFERENCES:}

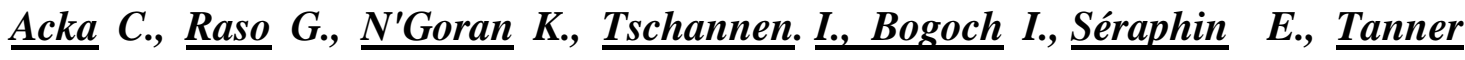
M., Obrist B. and Utzinger (2010): Parasitic Worms: Knowledge, Attitudes, and Practices in Western Côte d'Ivoire with Implications for Integrated Control, PLoSNegl Trop Dis; 4(12): 910. 
Amein N., El-Din S., Bayomi S. and Hossein Y. (2014): Knowledge and Practice of Caregivers about Intestinal Parasitic Infections at El-Minia City. Journal of education and practice; 5(3):642.

Ayalew A., Debebe T. and Alemayehu S. (2011):Prevalence and Risk Factors of Intestinal Parasites among Delgi School Children, North Gondar, Ethiopia Journal of Parasitology and Vector Biology; 3(5):75-81.

Azian N., San M., Gan Y., Yusri C., Nurulsyamz M., Zuhaizam A., Maslawaty H., Norparina M. and Vythilingam N. (2007): Malaysia Prevalence of Intestinal Protozoa in an Aborigine Community in Pahang, Tropical Biomedicine; 24(1):55-62.

Borg K.T. and Ryan R.G, (2010): Parasitic Disease, Ch 149, And P: 775-781 (http://www.cdc.gov/travel/).

Celiksoz A., Guler N., Guler G., Oztop Y. and Degerli S. (2005): Prevalence of Intestinal Parasites in Three Socioeconomically-Different Regions of Sivas, Turkey Journal of Health, Population and Nutrition; 23 (2):184-19.

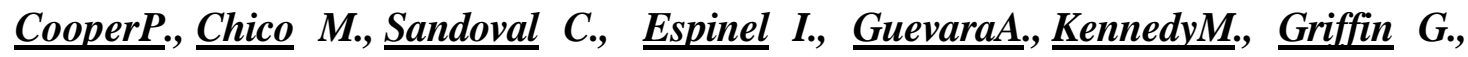
andNutman T. (2000): Journal of Infectious Diseases; 182(4):1207-1213.

Curtale F., Pezzotti P., Sharbini A., Al-Maadat H., Ingrosso P., Saad Y. andBabille M. (1998): Knowledge, Perceptions and Behavior of Mothers Towards Intestinal Helminths in Upper Egypt: Implications for Control Health Policy and Planning; Oxford University Press; 13(4):423-432.

El-Masry H.M., Ahmed Y.A., Hassan A.A., Zaky S., Abd-Allah E.S., El-Moselhy E.A., Baraka Y.A. and Abdel-Rahem M.A. (2007): Prevalence, Risk Factors and Impact of Schistosomal and Intestinal Parasitic Infections among Rural School Children in Sohag Governorate. The Egyptian Journal of Hospital Medicine; 11(29):616-630.

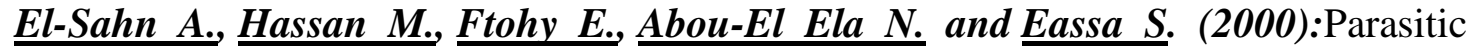
Infections and Maternal Awareness of Preschool Children in Karmouz District, Alexandria. Egypt Public Health Assoc; 75(1-2):1-29.

Espino JM., Espejo SR., Mendoza GV., Carreón AB. and Hoyos RC. (2011): Intervention to Prevent Intestinal Parasitic Reinfections among Tarahumara Indigenous School Children in Northern Mexico; 30(3):53.

Fitzgerald F . (2015): Primary, Secondary and Tertiary Prevention. Important in Certification and Practice, Health Education Associates.Nursing Volume, 12,(2): 7987.

Gross D. and Grady J. (2002): Group Based Parent Training for Preventing Mental Health Disorders In Children. Issues in Mental Health Nursing: 4(23) 3:367-383. 


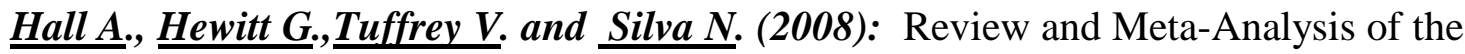
Impact of Intestinal Worms on Child Grow and Nutrition. Centre for Public Health Nutrition, University of Westminster, London, UK. Maternal \& Child Nutrition; 4(1):118-236.

Hazratitappeh, Kh., Mohammadzadeh., H., Nejad Rahim, R., Barazesh, A., Khashaveh., Sh,Taherkhani (2010):Prevalence of Intestinal Parasitic Infections among Mentally Disabled Children and Adults of Urmia, Iran, Dept.Of Parasitology \& Mycology, Faculty of Medicine, Urmia University of Medical Sciences, Urmia, IranInfectious disease specialist, Dept. Of Infectious Disease, Taleghani Hospital, Urmia Medical University.ran J Parasitol.5(2): 60-64.

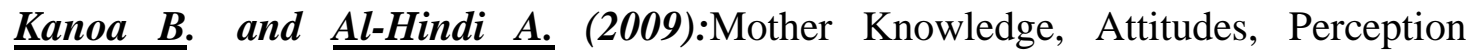
Regarding Intestinal Parasites and Diarrhea in Three Regions of Gaza Strip: Palestine. J Egypt SocParasitol; 39(3):827-36.

Karan A., Chapman G. and Galvani A. (2012): The Influence of Poverty and Culture on the Transmission of Parasitic Infections in Rural Nicaraguan Villages Hindawi Publishing Corporation. Journal of Parasitology Research; 20(12):12.

Mwambete D. and Kalison N. (2006):Prevalence of intestinal helminthic infections among underfives children and knowledge on helminthiases among mothers of the children in Dar Es-salaam, Tanzania. East African Journal of Public Heath, 3(1):8-11.

Remme J. (2007): Strategic Emphases for Tropical Diseases Research: A TDR Perspective. Trends Parasitol; 17(18): 421-6.

Savage AR. (2002): Providing Nursing Care for a Chagga Client of Tanzanic. J TranscultNurs; 13(3):248-53.

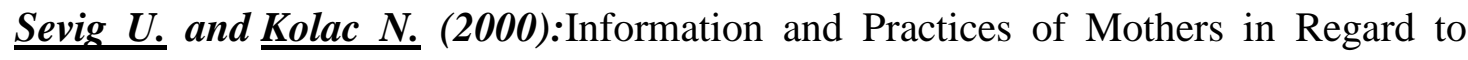
Intestinal Parasitic Infections, TürkiyeParazitolojiDergisi; 24 (2):164-168.

Tappe KH., ZadehHM., Khashaveh S., Rezapour B. and Barazesh A. (2008):Prevalence of Intestinal Parasitic Infections among Primary School Attending Students in Barandooz-Chayrural Region of Urmia, West Azerbaijan Province, Iran in. African Journal of Microbiology Research; 5(7):788-791.

Taylor M. (2008):Global Trends in Schistosomiasis Control World Health Organization. Bulletin of the World Health Organization. Geneva; 86 (10):738.

Tu XG., Chong ZX. and Bing Y. (2003): Evaluation of Intervention Strategy and Measures on the Control of Intestinal Parasitic Infections; 21(5):307-10. 


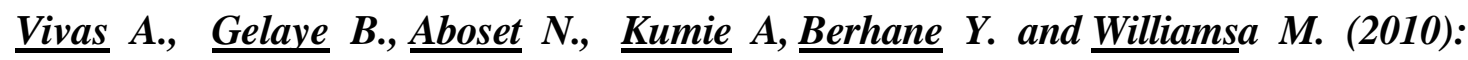
Knowledge, Attitudes, and Practices (KAP) of Hygiene among School Children in Angolela, Ethiopia J Prev Med Hyg; 51(2):73-79.

World Health Organization (WHO) (2007): Action against Worms. PPC Newsletter, Geneva: World Health Organization; 13(8):10.

W H O, (2000): Intestinal parasites available at http// / intpara/ burdens, htm.Accessed may 2000. 


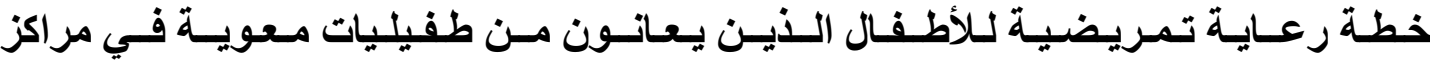 الـرعايـة الأوليـة فـي بورسبعيد}

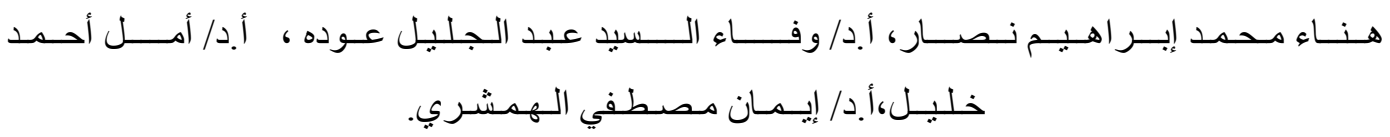

\section{الخلاصة}

عدوى الطفيليات المعوية بين الأطفال هي واحدة من أكثر المشاكل الملحة في هذه المرحلة الحرجة من الحباة. حيث

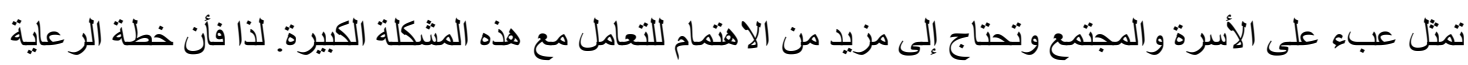
التمريضية والثقيف الصحي لهمادور هام في تعليم وتوجيه الأطفال والأمهات حول كيفيه الوقاية من الإصابة بالطفيليات المعوية .تهدف هذه الدراسة إلى تصميم وتنفيذ وتقييم تأثير خطة رعاية تمريضية للأطفال الذين يعانون من طفيليات معوية في مر اكز الرعاية الصحية الأولية في بورسعيد. واثتنلت عينة البحث علي 230 طفل في العينة التجريبية و

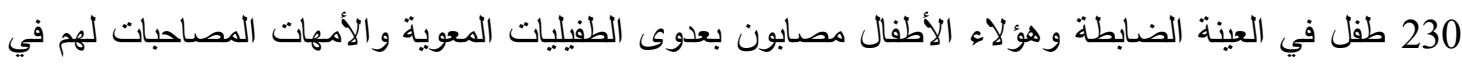
المر اكز الصحية التي أجريت فيها الدراسة وعددهم سبعة مر اكز للرعاية الصحية الاولية في مدينة بورسعيد. تم جمع

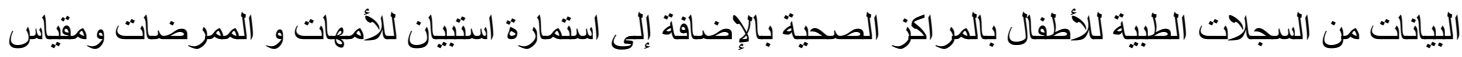
لأداء الطفل تجاه الطفيليات المعوية. وأثارت نتائج هذه الدراسة أن الانتاميبا والدودة الدبوسية أكثر أنواع العدوى الإصى انتثارا عند الأطفال في المجموعنين التجريبية والضابطة كما وجدا أن معلومات الأمهات عن الطفيليات المعوية قد الد الداه تحسنت بدرجة كبيرة عند المجمو عة التجرييية بعد تتفيذ خطة الرعاية التمريضية بالمقارنة بتحسن طفيف في معلومات

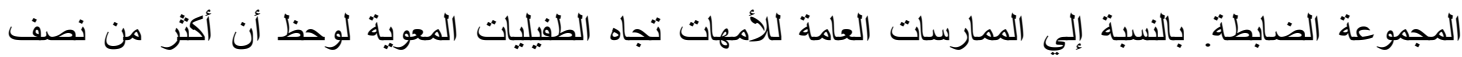
المجمو عتين التجرييية و الضابطة كانت ممارساتهم غير مرضية قبل تتفيذ خطة الرعاية التمريضية وعلي الجانب الآخر

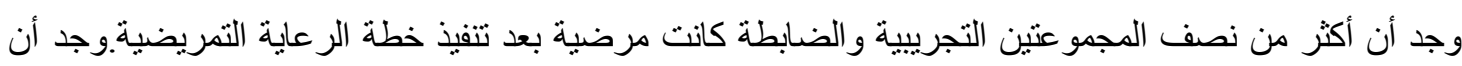
خطة الرعاية التمريضية لها دور هام في تعليم وتوجيه الأطفال والأمهات حول كيفيه الوقاية من الإصابة لهدية بالطفيليات المعوية ويوصى بعمل خطة رعاية تمريضية مبنية على الاحتياجات الفعلية المتعلقة بالأطفال الذين يعانون من طفيليات معوية. العمل على منع انتشار عدوى الطفيليات المعوية بين الاطفال وذللك عن طريق الاهتمام بالنظافة الثخصية و العامة .

الكلمات الدالة : خطة أدارة التمريض ، مرحلة ما قبل المدرسة للآطفال ، المعوية الطفيلية 